\title{
Complete Characterization of Novel MHMICs for V-Band Communication Systems
}

\author{
C. Hannachi, ${ }^{1}$ D. Hammou, ${ }^{1}$ T. Djerafi, ${ }^{1}$ Z. Ouardirhi, ${ }^{2}$ and S. O. Tatu ${ }^{1}$ \\ ${ }^{1}$ Institut National de la Recherche Scientifique, Centre Énergie, Matériaux et Télécommunications, \\ 800 de la Gauchetière Ouest, Montréal, QC, Canada H5A 1 K6 \\ ${ }^{2}$ Focus Microwaves, 1603 St. Regis, Dollard-des-Ormeaux, QC, Canada H9B 3 H7
}

Correspondence should be addressed to C. Hannachi; hannachi@emt.inrs.ca

Received 16 July 2013; Accepted 2 October 2013

Academic Editor: Alexander Koelpin

Copyright (C) 2013 C. Hannachi et al. This is an open access article distributed under the Creative Commons Attribution License, which permits unrestricted use, distribution, and reproduction in any medium, provided the original work is properly cited.

\begin{abstract}
This paper presents the characterization results of several new passive millimeter wave circuits integrated on very thin ceramic substrate. The work is focused on the design and characterization of a novel rounded Wilkinson power divider, a $90^{\circ}$ hybrid coupler, a rat-race coupler, and a novel six-port (multiport) circuit. Measurements show the wideband characteristics, allowing therefore their use for multi-Gb/s V-band wireless communication systems.
\end{abstract}

\section{Introduction}

The use of the $60-\mathrm{GHz}$ band has attracted a great deal of interest over the last few decades, especially for its use in future compact transceivers dedicated to high-speed wireless applications in indoor environments $(57-64 \mathrm{GHz})$ [1-3]. In this context, intensive research has been done to further develop new millimeter wave components for high data rate wireless communications according to the IEEE 802.15.3c standard. As previously demonstrated, the six-port technology offers an excellent alternative to conventional receiver architectures, especially at millimeter wave frequencies [4-6].

Nowadays, there are few promising high-quality fabrication technologies, yielding potentially low-cost millimeter wave components, such as the monolithic microwave integrated Circuit (MMIC) on GaAs or SiGe for large-scale production, and the miniature hybrid microwave integrated circuit (MHMIC) technology on very thin ceramic substrates, for small-scale production and prototyping $[7,8]$.

Moreover, several technologies have been intensively used for the millimeter wave circuit design and in-house prototype fabrication. We particularly note the coplanar, the substrate integrated waveguide (SIW), and the microstrip technology. The coplanar technology assures high-quality component design but is not well suited for low-cost production due to the difficulties in automating wire-bonding implementation, necessary for obtaining repeatable performances. On the other hand, the SIW technology assures highquality component design on thin ceramics [9] or the design of optimal transitions from planar to standard rectangular waveguides [10]. For further circuit miniaturization, the microstrip technology on very thin, high relative permittivity substrate is recommended.

As known, the microstrip line width is related to the characteristic impedance, substrate relative permittivity, and its thickness. It is to be noted that, due to reduced guided wavelength in high permittivity ceramic substrates, in order to keep the required circuit aspect ratio (guided wavelength versus the line width), the substrate must be as thin as possible. The optimal choice for frequencies greater than $60 \mathrm{GHz}$ is the $127 \mu \mathrm{m}$ thick alumina substrate, which is also easily compatible with the usual $100 \mu \mathrm{m}$ thick MMIC active components, to be integrated with planar passive MHMICs. The MMIC chips are placed in rectangular cuts on ceramics, on the top of the same metallic fixture, allowing thermal dissipation and easy wire bonding with MHMIC components, which are practically at the same height.

Initial designs and circuit characterization results of several MHMIC passive circuits on very thin ceramic substrate, designed for advanced millimeter wave systems operating in 60-90 GHz band, have been published few years ago [11]. 
This paper presents novel circuit designs, together with major improvements obtained in fabrication and characterization process in recent years.

\section{Calibration Techniques and Standards}

Measurement performance mainly depends on the accuracy of the calibration technique and its standards used for correcting the imperfections of the measurement system. These imperfections depend on several factors such as nonideal nature of cables and probes and the internal characteristics of the vector network analyzer (VNA) itself. In order to simplify calibration procedures and to obtain more accurate and reliable measurement by introducing much smaller systematic errors, the on-wafer calibration and measurement with picoprobes were adopted.

Typically, on-wafer calibration standards are fabricated either on the wafer including the device under test (DUT) or on a separate impedance standard substrate (ISS). The reference plane is usually taken at the probe tips. Nevertheless, for the DUT measurement in microstrip technology, onwafer standards fabricated on the same wafer as the DUT are required since the probe-to-standard transition can be designed to be very similar to the transition to the DUT. It sometimes happens that the transition between the probe tips and the coplanar line end is not well matched and parasitic and some wave modes occur at the contact of the probe tips. By taking the probe tips as measurement reference plane, the errors due to this transition are not corrected and may affect the measurement results.

Different calibration procedures or standards have been used for measuring microstrip-based circuits; among the most commonly used are line-reflect-match (LRM), lineline-reflect-match (LLRM), and thru-reflect-line (TRL) [12].

One of the most robust and popular technique is the TRL calibration, which is well suited to the on-wafer measurements at millimeter wave frequencies. According to previous comments, the reference plane is considered at the middle of the thru line.

The TRL calibration was done using on-wafer microstrip structures and the TRL algorithm supported by our vector network analyzer E8362B of Agilent Technologies.

A nonzero length thru is used to extend the reference plane a physical distance of $2286 \mu \mathrm{m}$ into the microstrip line in order to ensure direct measurement at the desired reference plane of the device, eliminating further deembedding and its associated uncertainties.

One microstrip delay line of $477 \mu \mathrm{m}$ length is used to cover the whole considered frequency band. Generally, in order to avoid phase uncertainties, for TRL calibration, the electrical length of the line standard is maximum $180^{\circ}$ at the highest operating frequency.

For the reflect standard, the designer can choose between the open and the short. In our opinion, the open standard is a better option at millimeter wave frequencies, due to the complex nature of the short-circuit design having repeatable performances in microstrip technology, especially when viaholes are used.

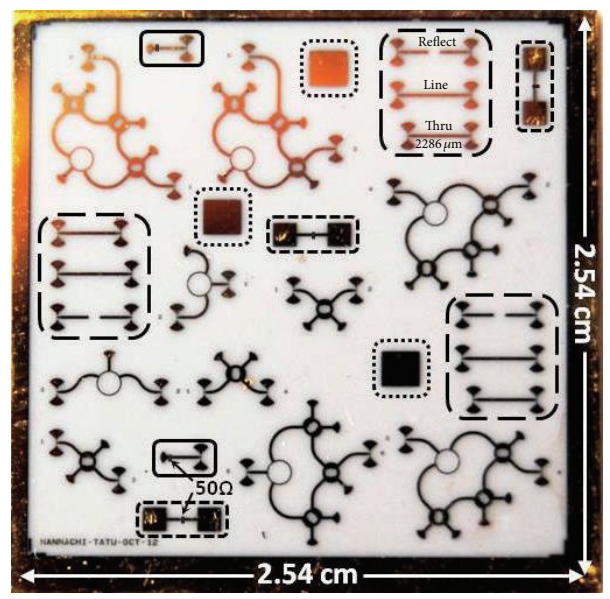

- - TRL calibration kits --- $50 \Omega$ test resistors
Microstrip conductors areas — Loads terminations $50 \Omega$
FIGURE 1: Microphotograph of the fabricated circuits on thin ceramic substrate of $2.54 \mathrm{~cm} \times 2.54 \mathrm{~cm}$.

In our designs, millimeter wave RF short circuits are implemented with quarter wavelength sectors, avoiding viaholes.

Figure 1 shows a microphotograph of typical fabricated circuits, including several identical TRL standard calibration kits. In order to optimize the fabrication cost and the measurement time, a maximum number of circuits to be characterized are fabricated on each ceramic die of $2.54 \mathrm{~cm} \times$ $2.54 \mathrm{~cm}$.

Due to the vulnerability of the very thin gold layer metallization $(1 \mu \mathrm{m})$, multiple identical kits have been fabricated in order to ensure successful calibration before each measurement. Small microstrip conductor areas were also added on die in order the properly align the picoprobes before measurement. In addition, $50 \Omega$ microstrip terminations and resistors test kits were used to verify the required value of $100 \Omega$ per square for the integrated loads.

Typical measurement results over $60-90 \mathrm{GHz}$ band of a microstrip line, after calibration, show matching results better than $-50 \mathrm{~dB}$ at both ports and a quasi-perfect transmission of $0 \mathrm{~dB}$ (with no more than $0.5 \mathrm{~dB}$ ripple, the intrinsic error of the VNA) when the picoprobes are properly aligned and positioned.

\section{Basic Circuit Characterization}

In order to integrate complete millimeter wave front-ends on ceramic substrates, the first step is to design basic circuits, such as couplers and power dividers/combiners. These components will be further utilized in antenna array and six-port downconverter or direct modulator designs.

As mentioned, the MHMICs have been designed and fabricated on a very thin ceramic substrate having a relative permittivity of 9.9 and a thickness of $127 \mu \mathrm{m}$. Advanced Design System (ADS) version 2011.05 of Agilent Technologies was used for circuits design and simulation. 


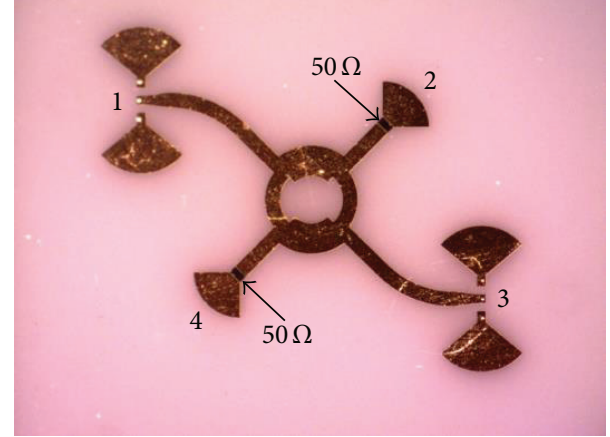

FIgURE 2: Microphotograph of the branch-line coupler.

In order to perform on-wafer measurement of the $S$ parameters, several circuits have been integrated in the same alumina substrate of $2.54 \mathrm{~cm} \times 2.54 \mathrm{~cm}$ size, as seen in Figure 1.

The symmetry of circuits is used to reduce the number of fabricated circuits required for complete characterization. For example, the full characterization of four port couplers requires minimum three different circuits due to the twodimensional symmetry. Only two circuits are needed for the Wilkinson power divider, because of its one-dimensional symmetry. Finally, five circuits are requested for the full characterization of our six-port design. All these circuits can be easily identified in Figure 1. The unused ports are connected to integrated $50 \Omega$ loads.

Even if the allowed frequency band starts from $57 \mathrm{GHz}$, all circuits are measured from $60 \mathrm{GHz}$ because of measurement set-up capabilities (WR-12 rectangular waveguides modules for the $60-90 \mathrm{GHz}$ millimeter wave extension of the VNA). However, the results can be extrapolated in the $57-60 \mathrm{GHz}$ band by symmetry and comparison with electromagnetic simulations.

3.1. $90^{\circ}$ Hybrid Coupler. Figure 2 shows the microphotograph of the $90^{\circ}$ hybrid coupler, prepared for port 1 to port 3 measurements.

As usual in our millimeter wave designs, the shape of the circuit is rounded, ensuring better $S$ parameter performances. The microstrip line widths are $126 \mu \mathrm{m}$ for the $50 \Omega$ microstrip line, $100 \mu \mathrm{m}$ for central line of the coplanar input, and $250 \mu \mathrm{m}$ for $50 / \sqrt{2} \Omega$ quarter wave line inside the coupler. The diameter of the coupler, measured between the centers of diametrically opposite microstrip lines, is around $625 \mu \mathrm{m}$.

As explained earlier, all via-holes are replaced with wideband RF short circuits. Details, such as $50 \Omega$ integrated resistor or the trace of the picoprobes on gold layer metallization after measurement, can be seen in the picture.

Figures 3 to 5 show measurement results for $S$ parameter magnitudes and phases of the circuit. Measurements are performed for $S_{21}, S_{31}$, and $S_{41}$ on three different circuits (see Figure 1, the secondary diagonal).

As seen in Figure 3, the measured return losses are better than $26 \mathrm{~dB}$ at $60 \mathrm{GHz}$ and better than $14 \mathrm{~dB}$ at the highest

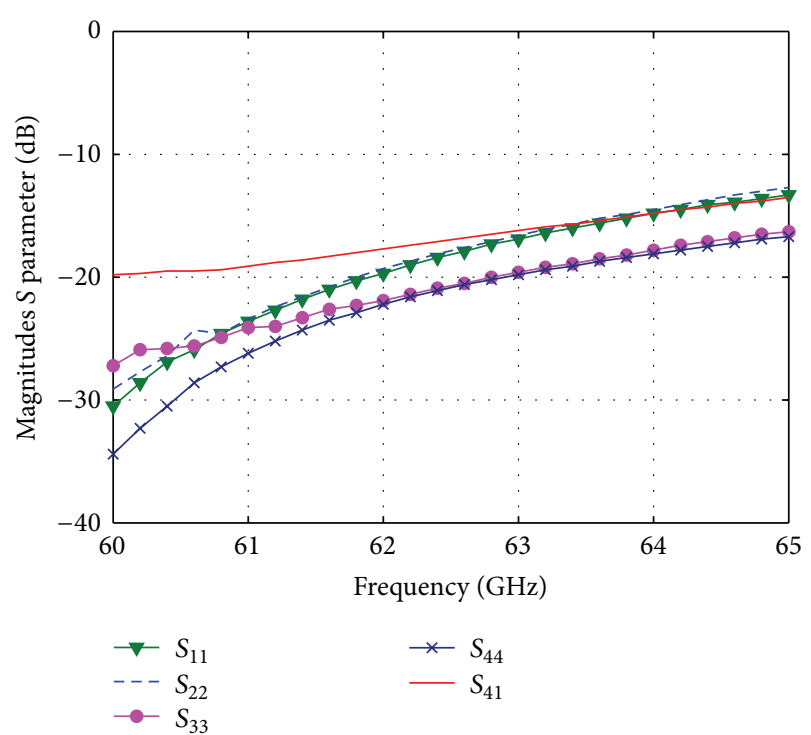

FIGURE 3: Measured input return loss for the $90^{\circ}$ hybrid coupler.

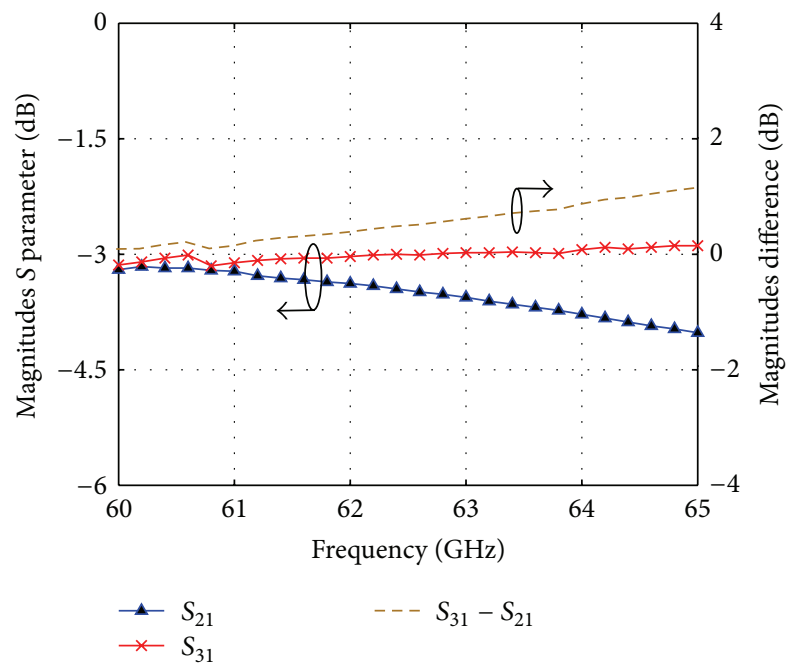

FIGURE 4: Measured transmission $S$ parameter magnitudes for the $90^{\circ}$ hybrid coupler.

frequency allowed for $\mathrm{V}$-band communications, $64 \mathrm{GHz}$. The isolation, $S_{41}$, is around $20 \mathrm{~dB}$ at $60 \mathrm{GHz}$ and has comparable values with return losses from $63 \mathrm{GHz}$.

The measured transmitted power is well splitted between the two outputs, especially around the central frequency allowed for V-band communications $(60.5 \mathrm{GHz})$. The magnitude unbalancing is practically zero at $60 \mathrm{GHz}$ and less than $1 \mathrm{~dB}$ at $64 \mathrm{GHz}$, as can be seen in Figure 4 .

Figure 5 shows that the phase difference between the two outputs is around $89^{\circ} \pm 1^{\circ}$ from 60 to $65 \mathrm{GHz}$.

Because in a six-port circuit the signal path crosses over two such couplers, these low values of magnitudes and phases unbalances are considered appropriate for modulation/demodulation schemes having up to 16 symbols. 


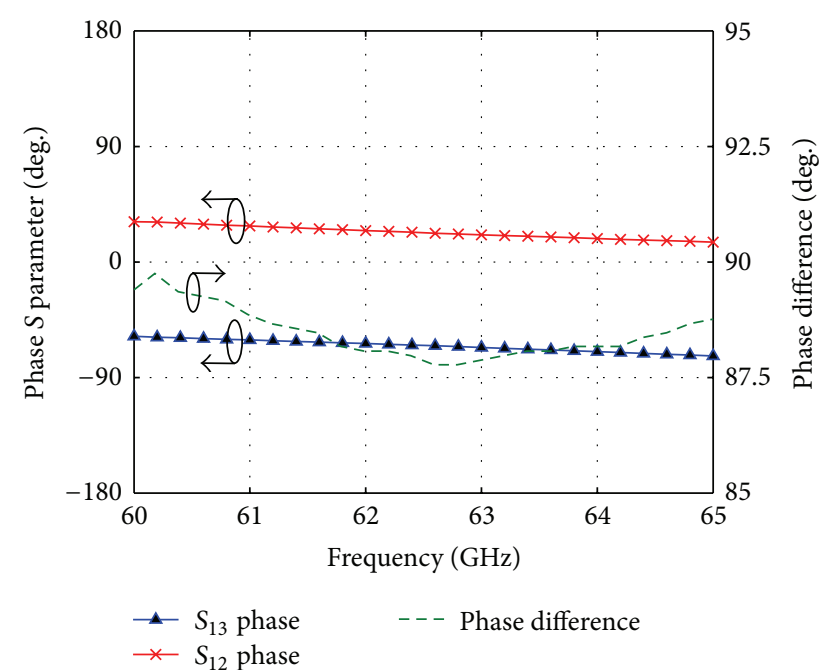

FIGURE 5: Measured transmission $S$ parameter phase difference for the $90^{\circ}$ hybrid coupler.

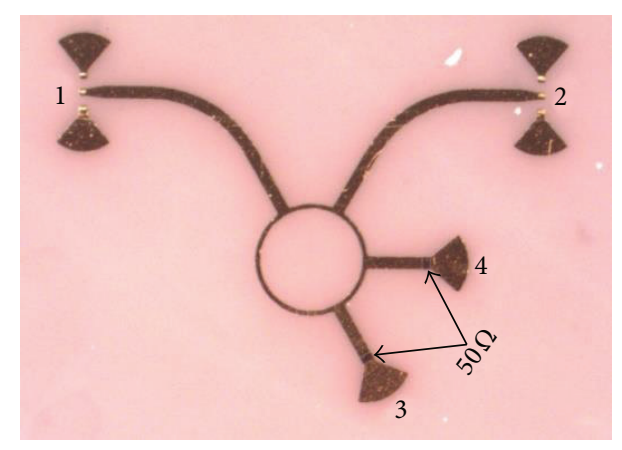

FIGURE 6: Microphotograph of the rat-race coupler.

3.2. Rat-Race Coupler. A rate-race coupler has been also designed and fabricated on a separate die, similar to that illustrated in Figure 1, along with other circuits. Multiple circuits have been fabricated to measure complete $S$ parameters of the coupler. As for the previous case, the unused ports are connected to integrated $50 \Omega$ loads.

Figure 6 shows the microphotograph of this rat-race coupler, prepared for port 1 to port 2 measurements.

In order to have a better idea of circuit size, let us see the same dimensions: the line widths are $126 \mu \mathrm{m}$ for $50 \Omega$ microstrip lines and $55 \mu \mathrm{m}$ for $50 \sqrt{2} \Omega$ characteristical impedance of the circular shape. The coupler circumference is equal to six quarter wavelengths and its diameter is $740 \mu \mathrm{m}$. Two integrated $50 \Omega$ millimeter wave loads are connected to ports 3 and 4 . Similar details, as in the previous circuit case, are visible on the picture: the $50 \Omega$ integrated resistors connected at unused ports and the traces of the picoprobes on gold layer metallization after measurement.

Figures 7 to 9 show measurement results for $S$ parameter magnitudes and phases of this rat-race coupler.

Figure 7 shows that the measured input return loss and isolation values are better than $15 \mathrm{~dB}$ over the whole considered frequency band, from 60 to $65 \mathrm{GHz}$. The isolation

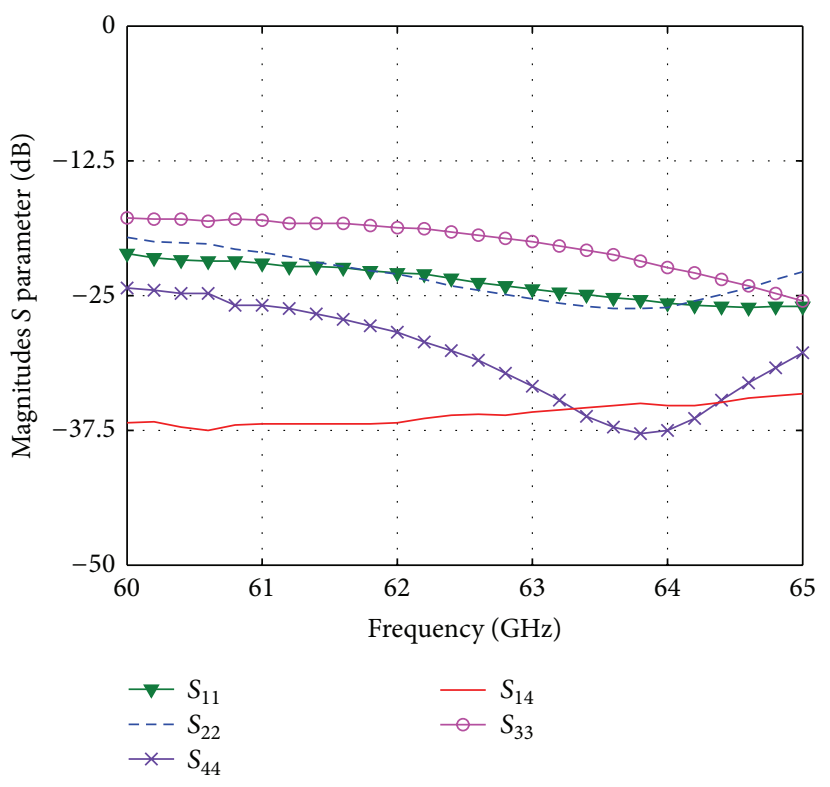

FIGURE 7: Measured input return loss for the rat-race coupler.

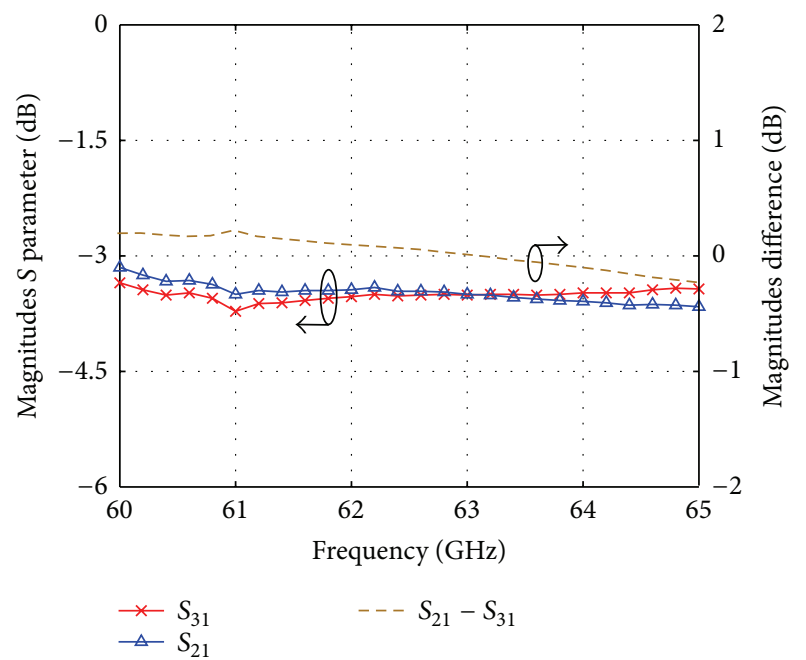

FIGURE 8: Measured transmission $S$ parameter magnitudes for the rat-race coupler.

$S_{14}$ reaches an exceptional value, of at least $35 \mathrm{~dB}$, due to the constant characteristic impedance over the ring. There are no steps in line width, which improves millimeter wave isolation.

As regards the transmitted power plotted in Figure 8, a quasi-equal split between the two outputs over the whole frequency range of interest is observed. The measured unbalancing is less than $0.5 \mathrm{~dB}$, comparable with the intrinsic error of the VNA.

Figure 9 shows the measurement of the transmission phase difference between the output ports. The phase difference value is equal to $180^{\circ}$, with a corresponding of phase error of $\pm 5^{\circ}$ over the considered frequency band. This error is close to $0^{\circ}$ at the central frequency allowed for V-band communications $(60.5 \mathrm{GHz})$. 


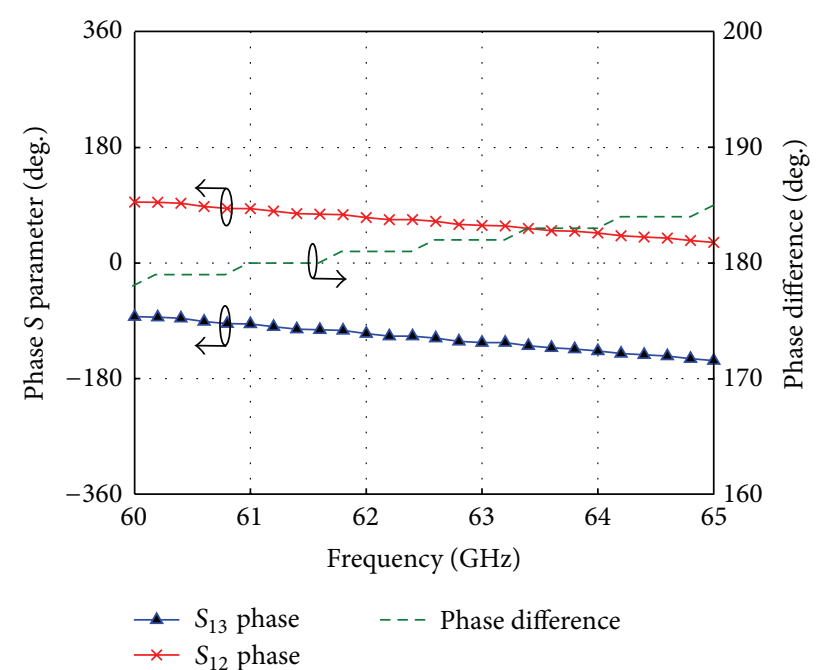

FIGURE 9: Measured transmission $S$ parameter phase difference for the rat-race coupler.

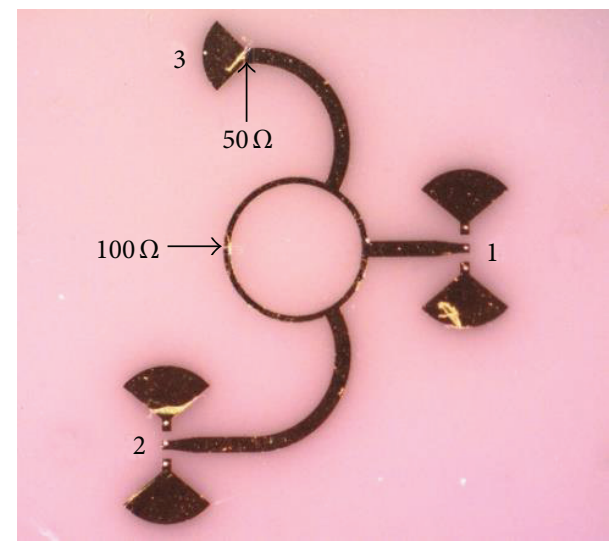

FIGURE 10: Microphotograph of the Wilkinson power divider/ combiner.

The rat-race coupler performances are ideal to design a V-band six-port-based downconverter using the quasiconventional architecture (with two pairs of antiparallel diodes connected to rat-race quadrature outputs), similar to those presented in [13] for automotive radars, at $77 \mathrm{GHz}$.

It is known that a conventional mixer uses such a ratrace coupler and a pair of antiparallel diodes. The six-port downconverter design is completed by adding a $90^{\circ}$ hybrid coupler to LO port and a Wilkinson at RF port and two diode mixers [14].

3.3. Wilkinson Power Divider/Combiner. Figure 10 shows the microphotograph of a novel rounded shape Wilkinson power divider [15] along with the transition from the coplanar wave to the microstrip line, requested for on wafer measurements. This circuit of Figure 10 is prepared for the port 2 to port 1 measurement. A second circuit, fabricated on the same ceramic die (see Figure 1), is used to measure isolation between ports 2 and 3 .

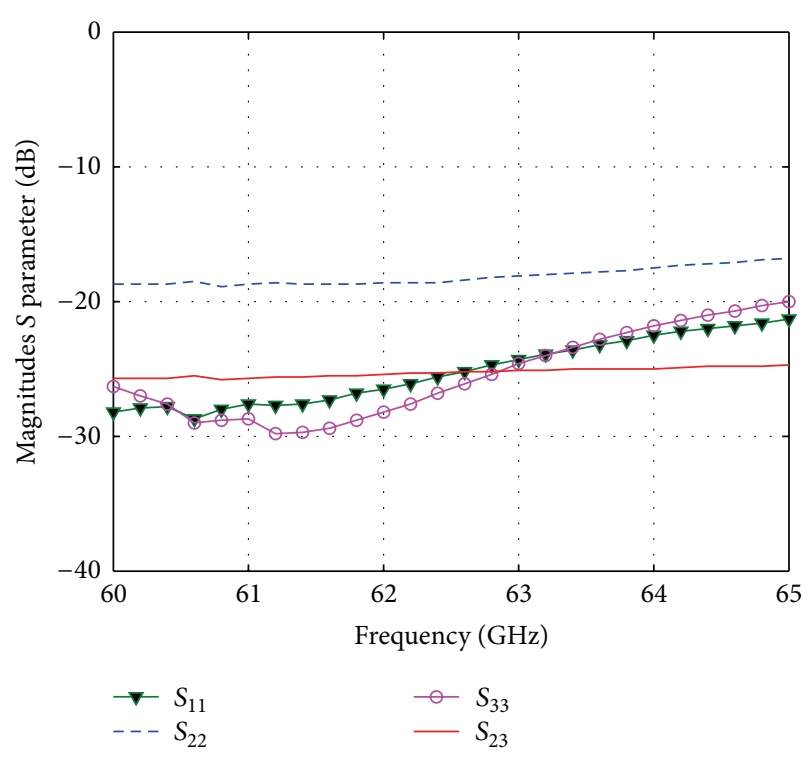

FIGURE 11: Measured return loss for the Wilkinson power divider/ combiner.

In order to avoid via holes, as in previous measurements, the $50 \Omega$ loads use a quarter wavelength open stub as millimeter wave RF short circuit (see port 3).

Figures 11 to 13 show measurement results of the novel Wilkinson power divider/combiner $S$ parameters (magnitude and phase).

As seen in Figure 11, the measured return losses have very good values. The port 2 return loss result is probably due to deviations from $50 \Omega$ values of the integrated load in the corresponding measurement circuit. However, due to the symmetry of the Wilkinson, the results must be closer to those obtained at port 3 using the second circuit. The isolation between two output/input ports of the divider/combiner, $S_{23}$, reaches also a very good value of around $25 \mathrm{~dB}$ over the whole band.

The power is almost equally split over the band, as illustrated in Figure 12; the magnitude unbalance is around $0.1 \mathrm{~dB}$.

The phase difference between the two outputs is less than $2^{\circ}$ over the considered band, as shown in Figure 13.

The glitches at $60.5 \mathrm{GHz}$ are due to an internal error of our VNA millimeter wave heads, which cannot be totally cancelled by calibration. It remains in the tolerance measurements of VNA, for both magnitude and phase measurements.

When the power divider circuit is part of a six-port, same conclusion as for previous circuits, the low values of magnitudes and phases unbalances are considered appropriate for the use of the six-port in modulation/demodulation schemes having up to 16 symbols.

3.4. Six-Port Circuit Characterization. Six-port (multiport) quadrature downconversion and direct modulation is an innovative approach in millimeter wave technology. A complete theory, validated by various simulations and measurements of V-band direct conversion receivers, has been published in recent years $[14,16]$. 


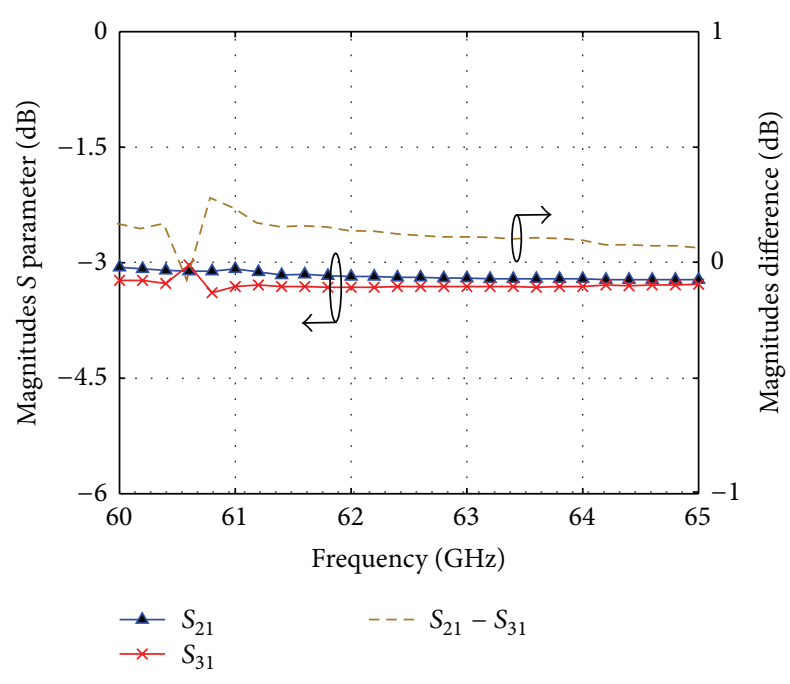

FIGURE 12: Measured transmission $S$ parameter magnitudes for the Wilkinson power divider/combiner.

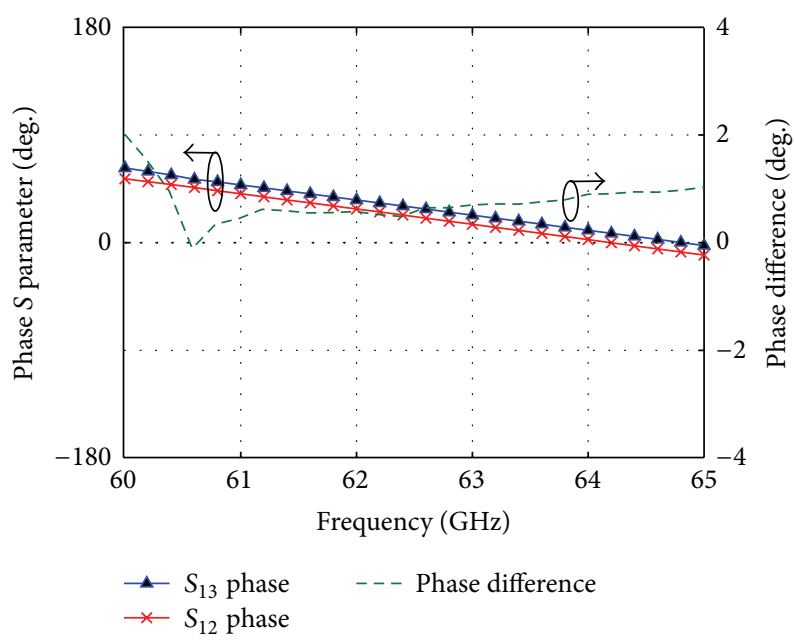

FIGURE 13: Measured transmission $S$ parameter phase difference for the Wilkinson power divider/combiner.

Figure 14 shows the block diagram of the proposed sixport, composed by a Wilkinson power divider and three $90^{\circ}$ hybrid couplers. As usually noted in all our previous publications, in a six-port downconverter, the port 6 is connected to the RF signal and the port 5 is connected to the LO signal; the other four output ports $(1,2,3$, and 4) are connected to power detectors [14].

In downconversion techniques, it has been demonstrated that the six-port technology allows improved results in terms of conversion loss and requires reduced LO power, as compared to the conventional methods (as low as -20 to $-25 \mathrm{dBm}$ to perform an efficient frequency conversion) [14]. On the other hand, a conventional diode mixer using antiparallel diodes acting at LO-driven switches requires around $+10 \mathrm{dBm}$ LO power for the same conversion loss. The excellent isolation between the six-port RF inputs is another important advantage versus the conventional approach [14].
A novel six-port circuit, having an improved symmetry and rounded shapes, has been designed using the novel Wilkinson power divider/combiner and the $90^{\circ}$ hybrid couplers presented in previous Sections 3.3 and 3.1, respectively. The central design frequency is $60.5 \mathrm{GHz}$, in the middle of allowed band of 57-64 GHz.

Measurements are performed, as explained for other circuits from $60 \mathrm{GHz}$, due to our measurement equipment capabilities. Once again, extrapolation of measurements and comparison with simulations help us to estimate the circuit behavior from 57 to $60 \mathrm{GHz}$.

The microphotograph in Figure 15 shows the six-port circuit prepared for port 2 to port 5 measurements. As requested, all other ports are terminated by adapted loads, integrated on the same substrate. The outer six-port dimensions are approximately $6.5 \mathrm{~mm} \times 6.5 \mathrm{~mm}$.

In order to measure the most important six-port $S$ parameters (such as RF-LO ports isolation $S_{65}$, input match at ports, power transmission between the RF and LO ports to the outputs, and the phase difference between transmitted signals), five six-port circuits have been integrated in the same alumina die, along with other basic circuits and required calibration standards (see Figure 1).

Figures 16 to 21 show some typical measurement results, according to the port numbers specified in Figures 14 and 15.

Figure 16 shows the measured return loss at port 6 (RF input) and port 5 (LO input) and isolation between them. At the central operating frequency of $\mathrm{V}$-band systems, all values are better than $20 \mathrm{~dB}$. In addition, the measured values are better than $15 \mathrm{~dB}$ at the highest frequency allowed for $\mathrm{V}$-band communications, $64 \mathrm{GHz}$.

Figure 17 shows the power splitting between the LO port and two adjacent output ports, $S_{25}$ and $S_{45}$. As compared to the theoretical value of $-6 \mathrm{~dB}$, very good results are obtained over the band. The supplementary insertion loss is around $1 \mathrm{~dB}$ at the central frequency and reaches $1.5 \mathrm{~dB}$ at the edge. The magnitude unbalance is close to $0 \mathrm{~dB}$ at $62.5 \mathrm{GHz}$ and less than $0.5 \mathrm{~dB}$ over the entire band.

The power splitting between the RF port (port 6) and two adjacent output ports, $S_{16}$ and $S_{36}$, is also shown in Figure 18. Good results have been obtained over the considered frequency band. These results show less than $1.5 \mathrm{~dB}$ of supplementary insertion loss, while the magnitude unbalance between requested ports (port 1 and port 3) does not exceed $0.6 \mathrm{~dB}$ in the whole frequency band of interest.

The measured return losses at output ports are illustrated in Figure 19. At the central operating frequency of V-band systems, all values are better than $25 \mathrm{~dB}$, keeping good values over the band.

Figure 20 shows the phase difference between the two typical transmission $S$ parameters, $S_{52}$ and $S_{54}$. It can be seen that the measured phase difference between these two outputs is close to the quadratic reference of $90^{\circ}$, as expected. The observed phase difference error is less than $\pm 2^{\circ}$ up to $64 \mathrm{GHz}$.

As regards the phase difference between the two typical transmission $S$ parameters, $S_{61}$ and $S_{63}$, depicted in Figure 21, it shows two quasi-parallel characteristics. The phase difference between two requested ports (port 1, port 3) is 


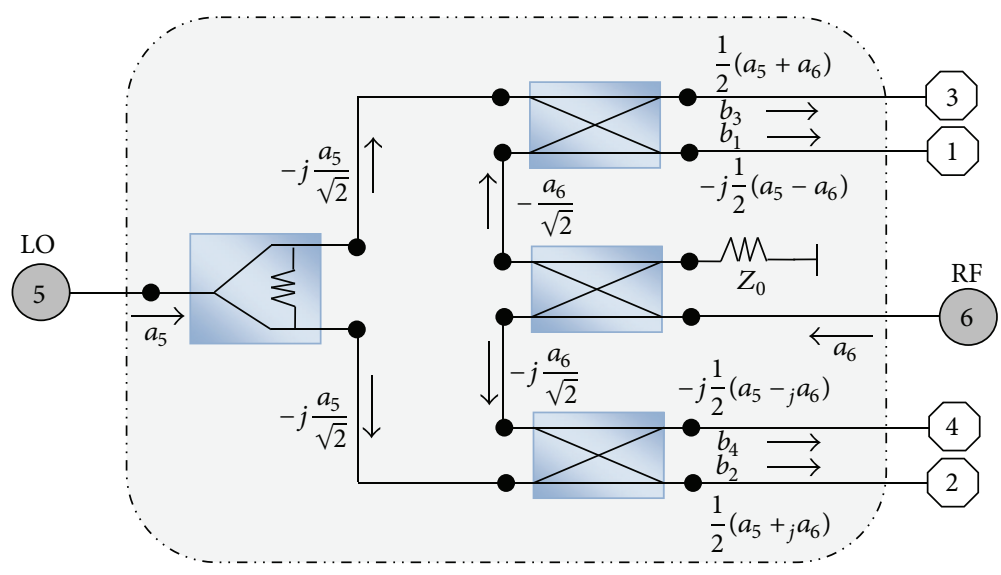

FIGURE 14: Six-port circuit block diagram.

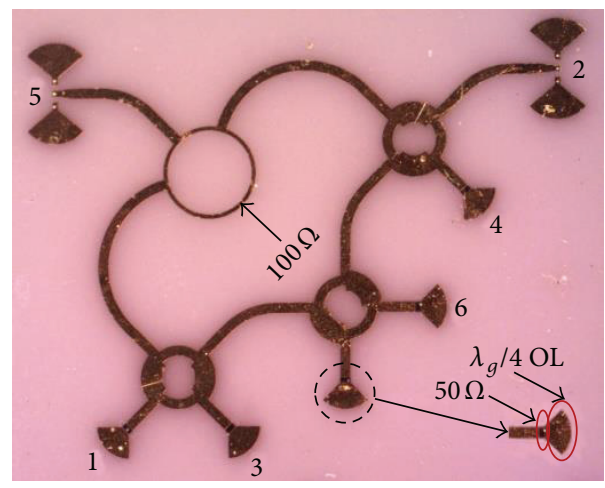

FIGURE 15: Microphotograph of the novel millimeter wave six-port circuit in a typical $S$ parameter measurement configuration.

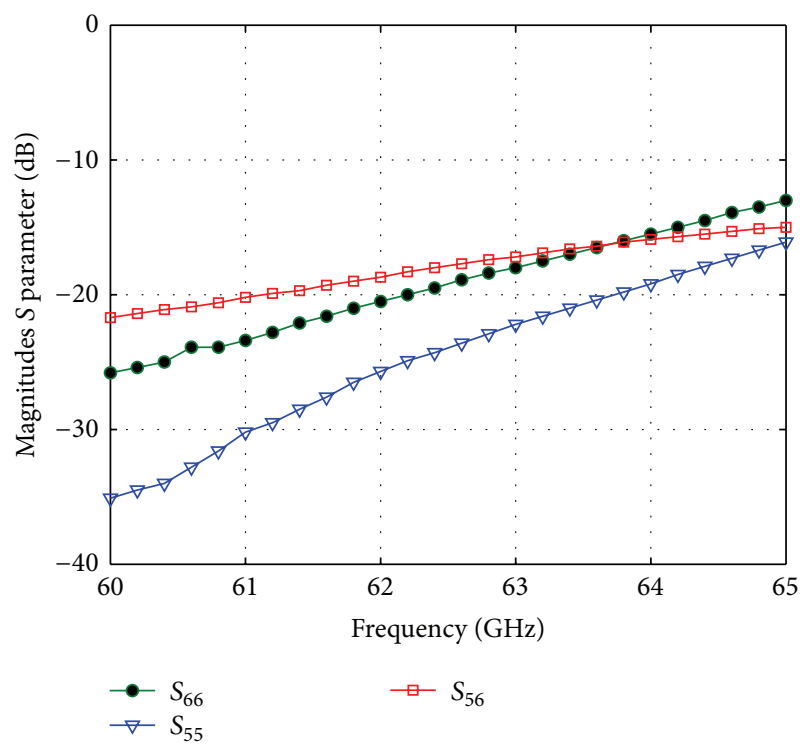

FIGURE 16: Measured RF inputs return loss and isolation for the proposed six-port circuit.

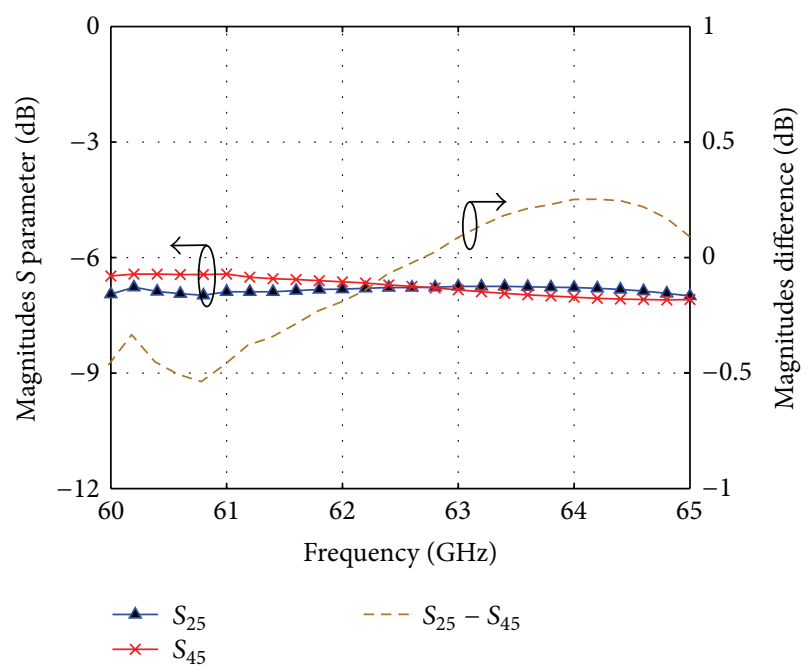

Figure 17: Typical measured transmission magnitudes $\left(S_{25}, S_{45}\right)$ for the proposed six-port circuit.

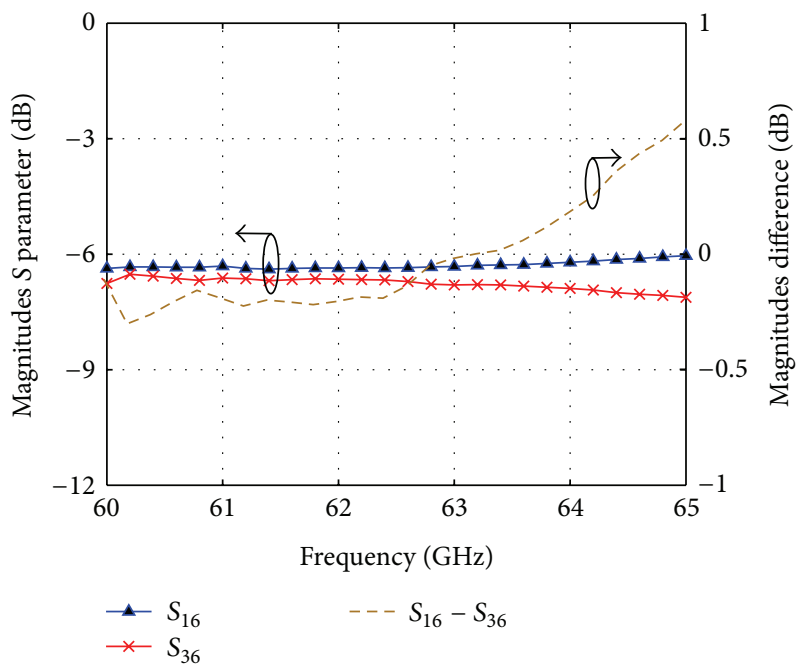

Figure 18: Typical measured transmission magnitudes $\left(S_{16}, S_{36}\right)$ for the proposed six-port circuit. 


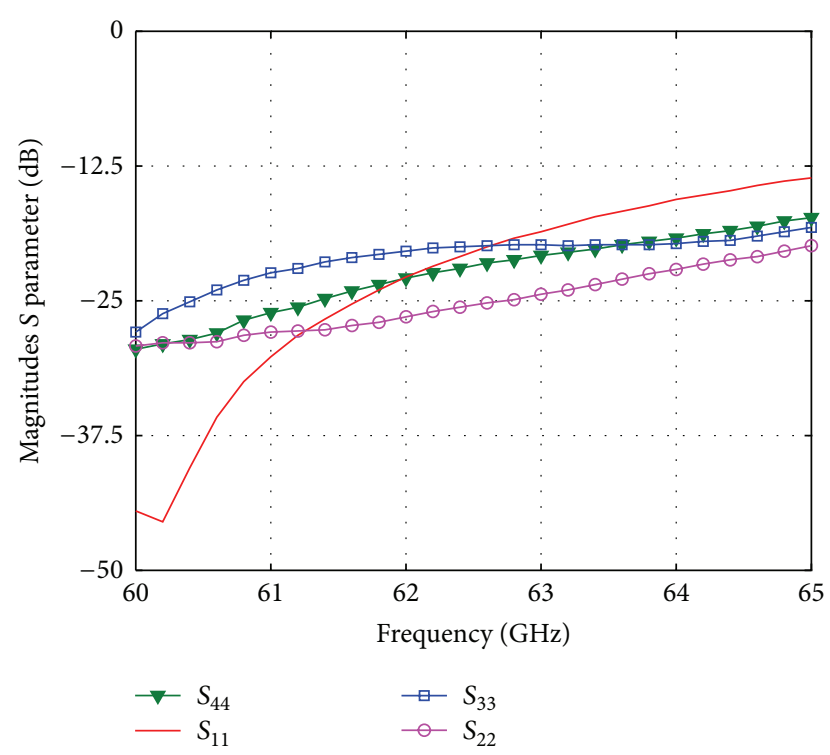

FIGURE 19: Typical measured outputs matches for the proposed sixport circuit.

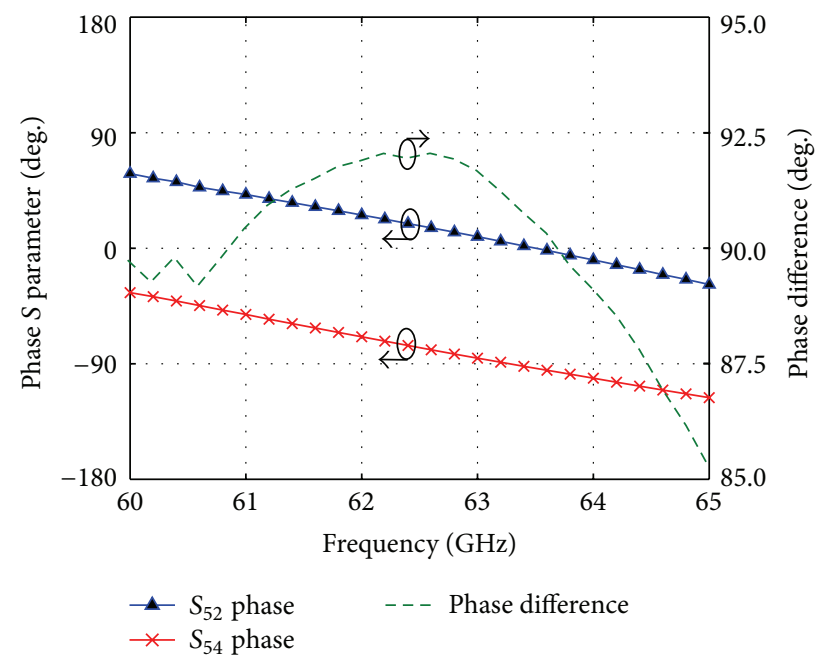

FIGURE 20: Typical measured transmission phase difference of $\left(S_{52}\right.$, $S_{54}$ ) for the proposed six-port circuit.

close to $90^{\circ}$. The phase difference error is approximately $3^{\circ}$ in the entire frequency band of interest.

\section{Conclusion}

Novel V-band MHMICs, including a rounded shape six-port circuit, have been presented in this paper. In order to improve circuits' performances, these MHMICs are fabricated in microstrip technology on very thin ceramic substrate.

Measurement results show that the proposed circuits are wideband components. The measured supplementary insertion losses, amplitude, and phase unbalancements are considered more than acceptable to build modulators/demodulators for modulation schemes having up to 16 symbols (BPSK to 16

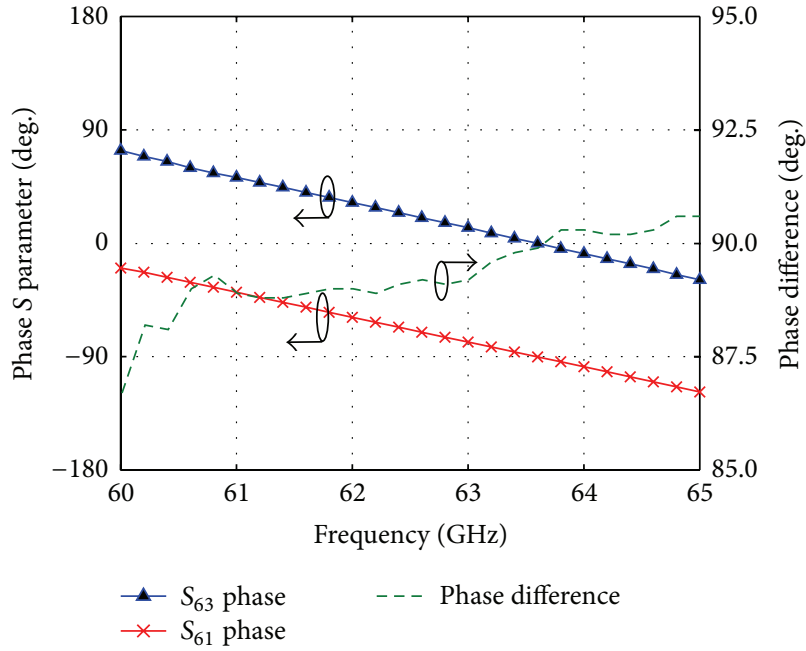

FIGURE 21: Typical measured transmission phase difference of $\left(S_{61}\right.$, $S_{63}$ ) for the proposed six-port circuit.

QAM, PSK, or dual star). Keeping in the account the $7 \mathrm{GHz}$ bandwidth allowed for V-band communication systems, the data-rates can reach quasi-optical values.

Six-port computer models have been implemented from the previous full-port measurements of Wilkinson and couplers. The two-port measurements of each circuit on die have been imported into ADS using data access components (DAC). Each model uses multiple DAC, interconnected according to the corresponding schematic of the six-port. The $S$ parameter simulation results, using the six-port model developed from these basic building boxes, agree with the measurements of the five six-port circuits presented in this paper. Therefore, the computer models will be considered for advanced system simulations of high-speed V-band wireless communication systems.

In conclusion, this new fabrication run has allowed us to improve the performances of the six-port circuit in order to be integrated in our future design of an entire millimeter wave front-end on a $2.54 \mathrm{~cm} \times 2.54 \mathrm{~cm}$ thin ceramic substrate. The die will integrate a $2 \times 8$ elements patch antenna array, a MMIC low noise amplifier, and a six-port quadrature downconverter.

\section{Conflict of Interests}

The authors declare that there is no conflict of interests regarding the publication of this paper.

\section{Acknowledgments}

The authors would like to acknowledge the support of the "Centre de Recherche en Électronique Radiofréquence" (CREER) of Montréal, funded by the "Fonds du recherché du Quebec-Natures and Technologies" (FRQNT), for the MHMICs fabrication. Manuscript received July 25, 2013. This work was supported in part by the National Science Engineering Research Council of Canada. 


\section{References}

[1] Federal Communications Commission, "Amendment of parts 2,15 and 97 of the commission's rules to permit use of radio frequencies above $40 \mathrm{GHz}$ for new radio applications," FCC 95499, ET Docket no. 94-124, RM-8308, December 1995.

[2] B. Laemmle, K. Schmalz, J. Borngraeber et al., "A fully integrated 120-GHz six-port receiver front-end in a 130-nm SiGe BiCMOS technology," in Proceedings of the IEEE Topical Meeting on Silicon Monolithic Integrated Circuits in RF Systems, pp. 129-131, Austin, Tex, USA, January 2013.

[3] P. Smulders, "Exploiting the $60 \mathrm{GHz}$ band for local wireless multimedia access: prospects and future directions," IEEE Communications Magazine, vol. 40, no. 1, pp. 140-147, 2002.

[4] A. Koelpin, G. Vinci, B. Laemmle, D. Kissinger, and R. Weigel, "The six-port in modern society," IEEE Microwave Magazine, vol. 11, no. 7, pp. 35-43, 2010.

[5] T. Eireiner, T. Schnurr, and T. Müller, "Integration of a six-port receiver for $\mathrm{mm}$-wave communication," in Proceedings of the IEEE Mediterranean Electrotechnical Conference (MELECON '06), pp. 371-376, Malaga, Spain, May 2006.

[6] B. Laemmle, K. Schmalz, C. Scheytt, D. Kissinger, and R. Weigel, "A $62 \mathrm{GHz}$ reflectometer for biomedical sensor readout in SiGe BiCMOS technology," in Proceedings of the 12th IEEE Topical Meeting on Silicon Monolithic Integrated Circuits in RF Systems, pp. 45-48, Santa Clara, Calif, USA, January 2012.

[7] W. Winkler, " $60 \mathrm{GHz}$ circuits in SiGe HBT technology," in Proceedings of the IEEE Compound Semiconductor Integrated Circuit Symposium (CSIC '05), pp. 109-112, Palm Springs, Calif, USA, November 2005.

[8] K. Leong and T. Itoh, "Advanced and intelligent RF front-end technology," in Proceedings of the IEEE Topical Conference on Wireless Communication Technology (WCT '03), pp. 190-193, Honolulu, Hawaii, USA, October 2003.

[9] E. Moldovan, S. O. Tatu, S. Affes, R. G. Bosisio, and K. Wu, "W-band substrate integrated waveguide radar sensor based on multi-port technology," in Proceedings of the 4th European Radar Conference (EURAD '07), pp. 174-177, Munich, Germany, October 2007.

[10] D. Hammou, M. Nedil, N. Kandil, E. Moldovan, and S. O. Tatu, "V-band millimeter-wave microstrip to rectangular waveguide transition," Microwave and Optical Technology Letters, vol. 55, no. 7, pp. 1696-1699, 2013.

[11] B. Boukari, D. Hammou, E. Moldovan, R. G. Bosisio, K. Wu, and S. O. Tatu, "MHMICs on ceramic substrate for advanced millimeter wave systems," in Proceedings of the IEEE MTT-S International Microwave Symposium (IMS '09), pp. 1025-1028, Boston, Mass, USA, June 2009.

[12] M. Nishimoto, M. Hamai, J. Laskar, and R. Lai, "On-wafer calibration techniques and applications at V-band," IEEE Microwave and Guided Wave Letters, vol. 4, no. 11, pp. 370-372, 1994.

[13] S. O. Tatu, B. Boukari, E. Moldovan, R. G. Bosisio, and K. $\mathrm{Wu}$, "Millimeter-wave multi-port radar sensor with integrated receiver front-end for automotive applications," in Proceedings of the IEEE MTT-S International Microwave Symposium, pp. 14, Montreal, Canada, June 2012.

[14] S. O. Tatu, E. Moldovan, and S. Affes, "Multi-port front-end and transceivers for V-band multi-gigabit/s communication systems," in Digital Front-End in Wireless Communications and Broadcasting Circuits and Signal Processing, pp. 707-732, Cambridge University Press, Cambridge, UK, 2011.
[15] D. Hammou, E. Moldovan, and S. O. Tatu, "Novel MHMIC millimeter wave power divider/combiner," in Proceedings of the Canadian Conference on Electrical and Computer Engineering (CCECE '11), pp. 280-283, Ontario, Canada, May 2011.

[16] T. Brabetz and V. Fusco, "Six-port receiver front-end MMIC for V-band MBS applications," in Proceedings of the 11th Gallium Arsenide Applications Symposium (GAAS '03), pp. 97-99, Munich, Germany, October 2003. 

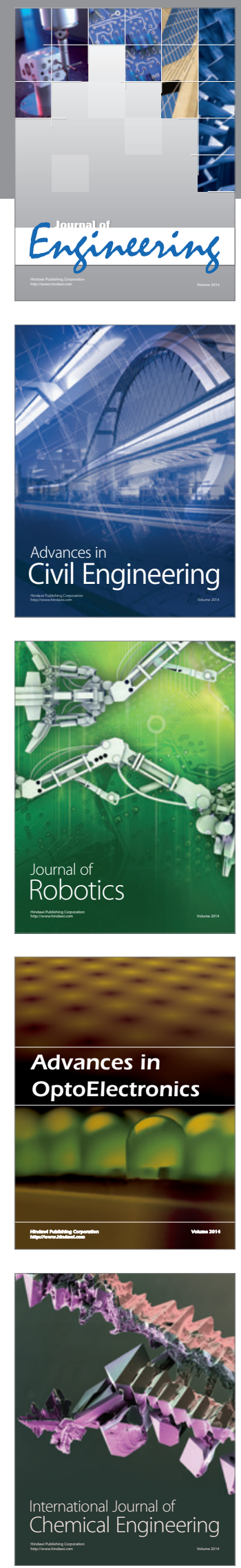

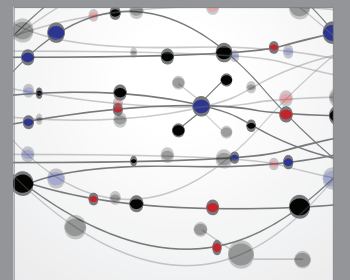

The Scientific World Journal
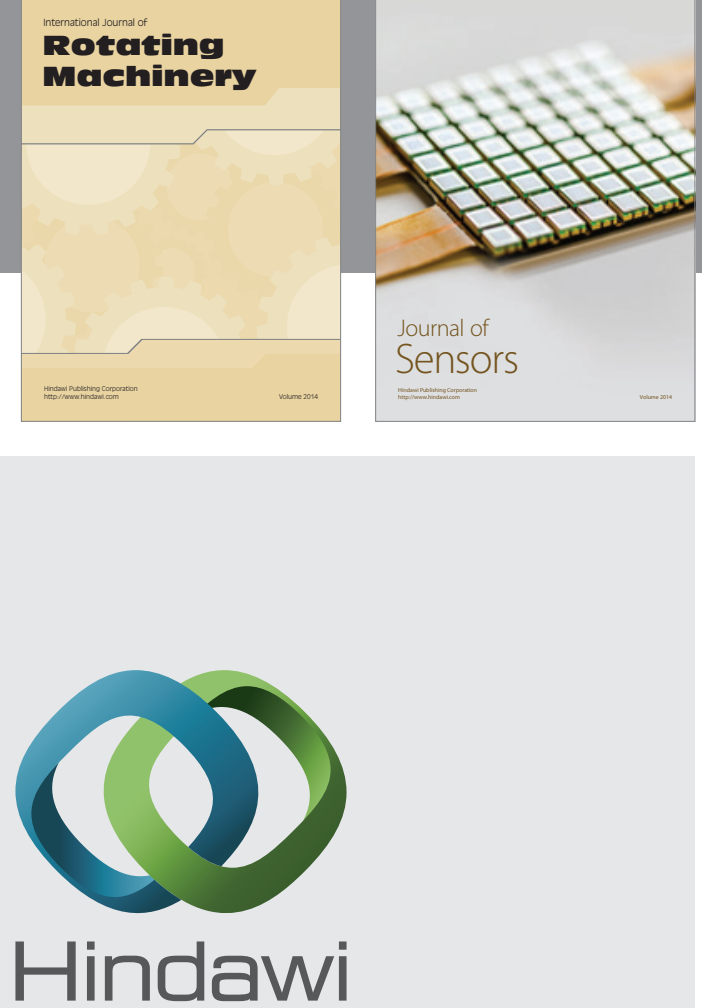

Submit your manuscripts at http://www.hindawi.com
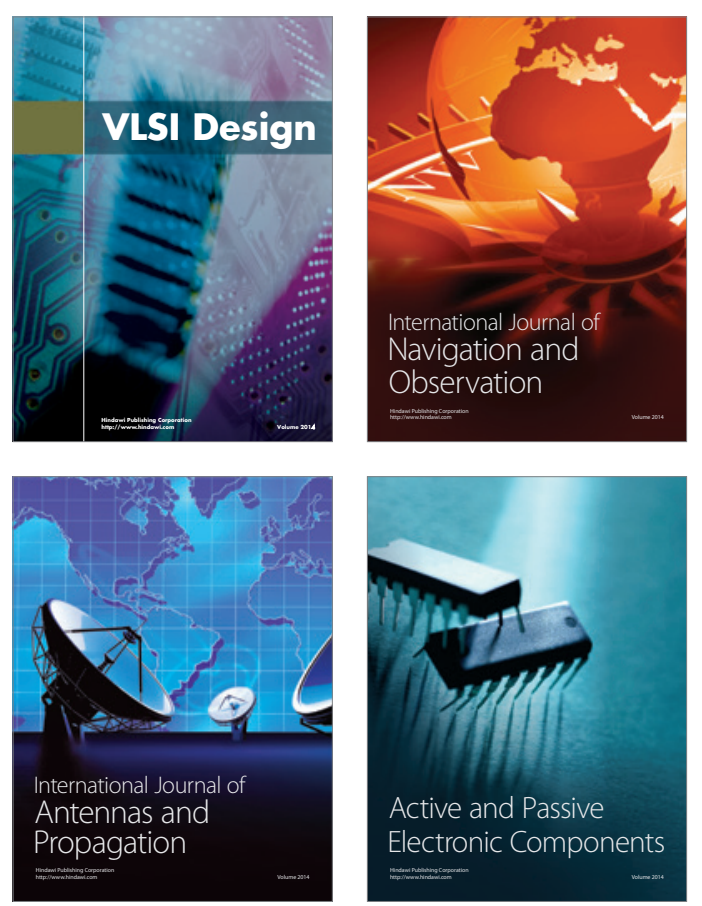
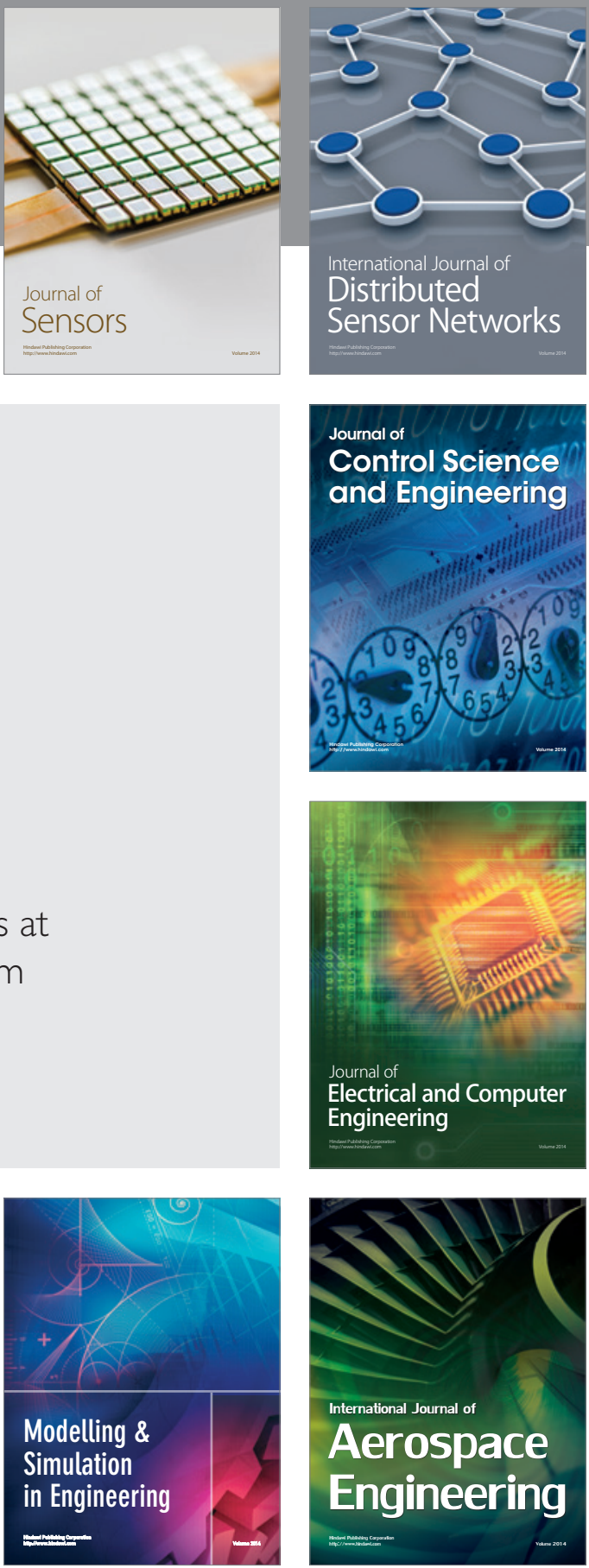

Journal of

Control Science

and Engineering
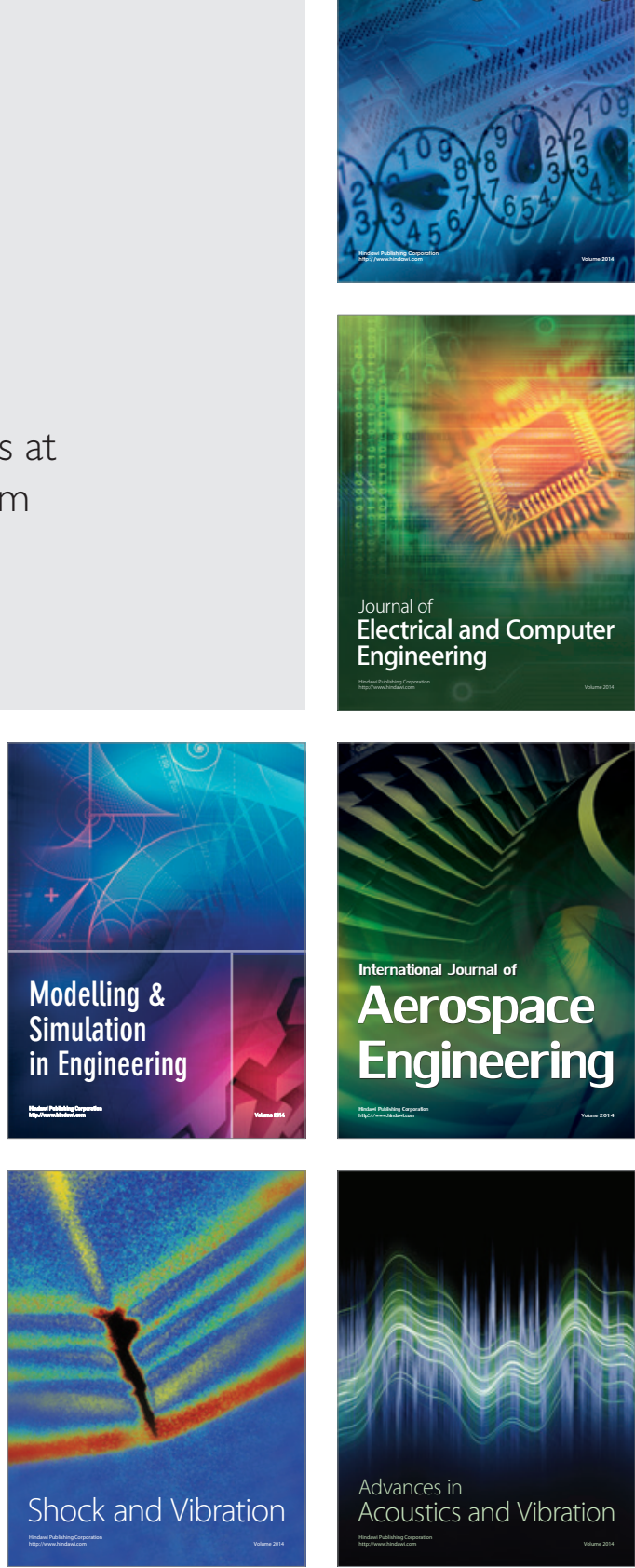